\title{
Fox Chase SCID Mouse
}

National Cancer Institute

\section{Source}

National Cancer Institute. Fox Chase SCID Mouse. NCI Thesaurus. Code C15158.

Discovered by Bosma (1980) at Fox Chase Cancer Center, the CB17 SCID mouse has an autosomal recessive mutation in the Prkdc gene which causes a severe combined immunodeficiency affecting B and T lymphocytes. 